\title{
Análisis de la responsabilidad social universitaria: un estudio comparativo en Latinoamérica
}

\author{
Blanca Estela Bernal Escoto* \\ María Paula Díaz Rojas ${ }^{* *}$
}

Recibido: 02 de septiembre de 2020

Aprobado: 28 de septiembre de 2020

Bernal, B., Díaz, P. (2020). Análisis de la responsabilidad social universitaria: un estudio comparativo en Latinoamérica. Revista Activos, 18(2), 111-135. https://doi.org/10.15332/25005278/6262

\section{Clasificación JEL: M14, P28}

\section{Resumen}

La presente investigación analiza las prácticas que han tomado las instituciones de educación superior de acuerdo con la responsabilidad social universitaria. Se plantea una investigación documental, con un seguimiento

\footnotetext{
* Profesora e investigadora de la Facultad de Contaduría y Administración de la Universidad Autónoma de Baja California.

Correo electrónico: blancab@uabc.edu.mx ORCID: https://orcid.org/0000-0002-8721-256

** Estudiante de la facultad de Contaduría Pública de la Universidad Santo Tomas. Correo electrónico: maria.diazr@usantotomas.edu.co ORCID: https://orcid.org/0000-0003-4055-4510
} 
a un enfoque medioambiental, por lo que se realiza un estudio comparativo con un sistema de indicadores para la medición de las áreas referentes a la protección del medio ambiente en dos universidades de Latinoamérica. El análisis de este estudio dio como resultado que las universidades participantes sí han aplicado medidas técnicas para el cuidado, manejo y compromiso con el medio ambiente; sin embargo, se les aconseja proponer nuevas políticas y estrategias para tener un mejor uso y cuidado del medio ambiente a nivel institucional.

Palabras clave: responsabilidad social universitaria, indicadores medioambientales, instituciones de educación superior.

\title{
Analysis of university social responsibility: a comparative study in Latin America
}

\begin{abstract}
This research analyzes the practices adopted by Higher Education Institutions (HEI) in accordance with university social responsibility (USR). A documentary research is proposed, with a follow-up to an environmental approach, for which a comparative study is carried out with a system of indicators for the measurement of the areas related to environmental protection in two Latin American universities. The analysis of this study showed that the participating universities have applied technical measures for the care, management and commitment to the environment; however, they are advised to propose new policies and strategies for a better use and care of the environment at institutional level.
\end{abstract}

Keywords: university social responsibility, environmental indicators, higher education institutions. 


\section{Introducción}

En la actualidad, la responsabilidad social universitaria (RsU) ha tenido un enfoque integral para definir la naturaleza de la enseñanza superior: con el fin de proporcionar una base que sustente el desarrollo humano y así obtener una formación y un aprendizaje óptimo, la Rsu ha generado la necesidad de desarrollar diferentes habilidades en contexto institucional. Tanto para las Instituciones de Educación Superior (IES), como para la RSU, el tema de educación ambiental es reciente, teniendo en cuenta que la responsabilidad social ambiental coincide con el desarrollo sostenible (Franco, et al., 2017).

Esta investigación se contextualiza en la propuesta metodológica formulada por Vallaeys, De la Cruz y Sasia (2009); por un lado, incorporando la información en torno a los procesos de gestión ética y ambiental; y, por otro lado, identificando los grupos de interés de la universidad, para formar ciudadanos responsables y solidarios, con una obtención y difusión de conocimientos sociales, además de una participación social con un desarrollo más humano y sostenible.

La RSU es un enfoque integral que busca encontrar soluciones innovadoras para atender las necesidades sociales de las IEs; su premisa gira en torno a la solidaridad, la equidad y la justicia social, garantizando a los actores en el proceso docente un desarrollo sostenible en sus grupos de interés. Esto significa que las instituciones educativas que deseen implementar esta iniciativa deben llevar un trabajo colaborativo (Vallaeys, s. f.). En ese contexto, y teniendo en cuenta que cada vez hay más IEs preocupadas por formar profesionistas responsables con su entorno, la sociedad y el medio ambiente, surgió la necesidad de realizar un estudio comparativo entre la Universidad Autónoma de Baja California, México, y la Universidad Santo Tomás de Colombia.

En este punto, cabe mencionar que la polémica sobre la responsabilidad social (Rs) surgió en la posguerra de la Segunda Guerra Mundial, debido a la violencia social y los crímenes de lesa humanidad cometidos por los gobiernos y sus líderes contra los contingentes de grandes poblaciones. Aunque 
en un principio se consideró una cuestión moral, para el comportamiento del sujeto, la responsabilidad moral sólo constituye un caso específico de Rs. Además de atribuirse tanto a los individuos como a las organizaciones, incluidas las universidades, también se configura en los planos económico, político y social (González, Méndez, García y Arguello, 2017).

La importancia de esta investigación radica en la información que se tiene acerca de la responsabilidad social universitaria en las IES; el objetivo es analizar si estas instituciones están trabajando el tema medioambiental en sus planes de estudio y cómo lo están ejecutando. Cabe resaltar que esta información será útil para resolver varias incógnitas que se tienen sobre el tema: ¿qué tanto estas universidades han implantado el tema medioambiental? y ¿cómo las universidades participantes se responsabilizan por los impactos medioambientales generados? Por consiguiente, este estudio sentará las bases para futuras investigaciones.

En cuanto a los objetivos del estudio, se pretende: primero, identificar los indicadores del impacto ambiental considerados por las IEs participantes en su modelo de Rsu; posterior a ello, conocer los lineamientos de la responsabilidad social universitaria en el ámbito medioambiental; y, por último, indagar sobre los enfoques ambientales que las universidades han venido implementando en la RSU.

El presente artículo de investigación tiene un valor social/medioambiental para las universidades que actualmente desean ser evaluadas como Instituciones de Educación Superior socialmente responsables, puesto que se proporcionan las mejores prácticas en el ámbito medioambiental, por parte de dos IEs reconocidas a nivel internacional, como son la Universidad Autónoma de Baja California en Tijuana, Baja California, México, y la Universidad de Santo Tomas en Bogotá, Colombia.

Finalmente, el aporte metodológico radica en la generación del conocimiento al realizar el primer estudio comparativo a nivel Latinoamérica; debido a que facilita a las IEs que deseen certificarse en Responsabilidad 
Social Universitaria los lineamientos necesarios para conseguirlo, así como los indicadores que deben ser atendidos.

\section{Marco teórico}

El concepto de responsabilidad social universitaria nace a partir de la noción de desarrollo sostenible, el cual surgió de la necesidad de introducir cambios en el sistema económico existente, basado en la máxima producción, el consumo, la explotación ilimitada de recursos y el beneficio como único criterio de la buena marcha económica.

En la década de 1970, Ignacy Sachs, consultor en las Naciones Unidas para temas de medio ambiente y desarrollo, propuso la palabra "ecodesarrollo" con el fin de conciliar el aumento de la producción que reclamaban con urgencia los países del tercer mundo, y el respeto a los ecosistemas que permitirían mantener las condiciones de la habitabilidad de la tierra (Larrouyet, 2015). Este término empezó a utilizarse en los círculos internacionales relacionados con el medioambiente y el desarrollo. Sin embargo, a pedido del entonces jefe de la diplomacia norteamericana, Henry Kissinger, quién desaprobó el término "ecodesarrollo", el presidente del Programa de las Naciones Unidas para el Medio Ambiente debió sustituir el término por "desarrollo sostenible", que los economistas más convencionales podían aceptar sin recelo, al confundirlo con el "desarrollo autosostenido".

Ahora bien, de conformidad con Bustos e Inciarte (2012), el concepto de RSU en las Instituciones de Educación Superior siempre ha existido bajo el nombre de "función social de las universidades". En Latinoamérica, los antecedentes del término se relacionan con los fines universitarios plasmados en el Congreso Internacional de Estudiantes Americanos llevado a cabo en 1908 en Montevideo, Uruguay; donde, además, surgió el término de extensión universitaria que constituyó la base para la Reforma de Córdoba de 1918.

Emitida en París en 1998, aprobada por la Conferencia Mundial sobre Educación Superior y avalada por la Organización de las Naciones Unidas 
para la Educación, la Ciencia y la Cultura, la Declaración Mundial sobre la Educación Superior para el Siglo XxI estableció que entre las misiones y valores de la educación superior se encuentran las de formar diplomados altamente cualificados que participen activa y responsablemente en la sociedad, construyendo un espacio abierto de formación a lo largo de la vida. Asimismo, dicha declaración establece una serie de valores que pueden entenderse como los antecedentes de la responsabilidad social universitaria (Ojeda y Álvarez, 2015).

Posteriormente, en los años ochenta, se presenta el informe Brundtland ante la Comisión Mundial para el Medio Ambiente y Desarrollo, en el cual se hace hincapié en un futuro común, siendo primordial el examen de los problemas más críticos en torno al desarrollo y el medioambiente. A partir de allí, se difunde el término de desarrollo sustentable, como "aquel que responde a las necesidades del presente de forma igualitaria, pero sin comprometer las posibilidades de sobrevivencia y prosperidad de las generaciones futuras" (Zepeda, Andrade, y González, 2017, pp. 36-37). En ese contexto, el concepto de desarrollo sustentable está constituido por tres facetas: económico, ecológico y social.

Por una parte, Estrella y González (2017) define el desarrollo sustentable como aquel que satisface las necesidades del presente sin comprometer las capacidades que tienen las futuras generaciones para satisfacer sus propias necesidades. Por otra parte, la Universidad Autónoma de Nuevo León (2014) explica que aquel

[...] es el proceso de cambio continuo de aplicación local, regional o global que responde a objetivos y metas universales de transformación social apropiada, para satisfacer las necesidades de bienes y servicios de una población o conjunto de poblaciones, por tiempo indefinido, sin degradar irreversiblemente la capacidad productiva de la tierra, el patrimonio natural y su habilidad para mantener la población de un lugar (pp. 178-179). 
Sumado a esto, Ordóñez y Werner (2019) afirman que el concepto de desarrollo sostenible aplicado a la educación tiene como finalidad proporcionar a toda la comunidad estudiantil la oportunidad de adquirir conocimientos, valores y modelos de comportamiento, necesarios para integrarse a la sociedad de forma positiva y perdurable.

Posteriormente Vallaeys (2014), a inicios del año 2000, acuñó la expresión responsabilidad social universitaria en América Latina, alrededor de los esfuerzos teóricos y prácticos de la red chilena Universidad Construye País y de la Red Latinoamericana de Universidades animada por la Iniciativa Interamericana de Ética, Capital Social y Desarrollo, promovida por el gobierno noruego en el seno del Banco Interamericano de Desarrollo.

Se habla mucho en los últimos tiempos de la "responsabilidad social" como de una dimensión ética que toda organización o institución debería tener como visión y promover en su actividad diaria. Hace buen tiempo que el mundo empresarial ha acogido y desarrollado la idea, pero la reflexión acerca de la responsabilidad social recién empieza a darse en el ámbito universitario (Vallaeys, 2014, p. 107).

En las últimas décadas se ha sumado otro concepto a la ecuación: la universidad socialmente responsable. Esta es concebida como

[...] aquella que procura desarrollar todos sus productos ya sea a nivel de investigación, vinculación con el medio o formación de profesionales en una real asimilación o coincidencia con lo que necesita la región o el territorio donde está asentada la universidad (directivo Universidad de Antofagasta) (Gaeta, 2015, pp. 97-99).

En la siguiente figura muestra la evolución del concepto de responsabilidad social universitaria desde sus orígenes. 
Figura 1. Evolución del término RSU

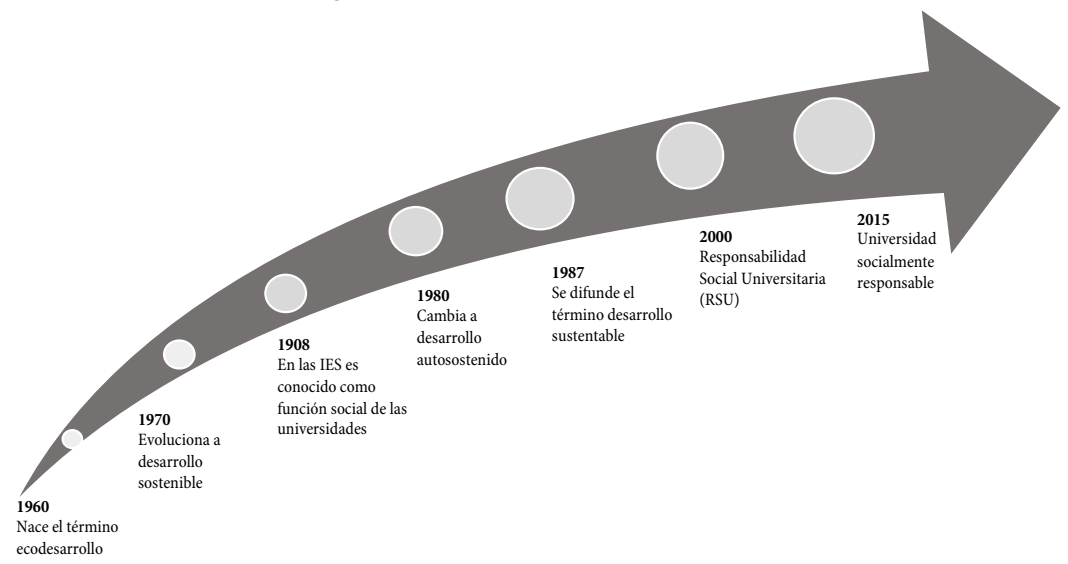

Fuente: Elaboración propia con información de Bustos e Inciarte (2012), Estrella y González (2017), Gaete (2015), Larrouyet (2015), Ojeda y Álvarez (2015), Vallaeys (2014), Zepeda, Andrade y Gonzalez (2017).

De acuerdo a Vallaeys, De la Cruz, y Sasia (2009)

[...] La exigencia ética de sostenibilidad invita a redefinir los modelos de gestión de las organizaciones y los territorios locales, nacionales y regionales. La exigencia de sostenibilidad ha conducido a los diversos movimientos de responsabilidad social a través del mundo a asumir poco a poco cuatro principios que definen el concepto a partir de las negativas:

a. La responsabilidad social no es acción social filantrópica al margen de la actividad principal de la organización, sino un nuevo sistema de gestión de la organización.

b. La responsabilidad social no es una moda pasajera, sino una obligación universal para asegurar la sostenibilidad social y ambiental de nuestro modo de producción y consumo en un planeta frágil en el cual todos tenemos iguales derechos a una vida digna. 
c. La responsabilidad social no es una función más de la organización, sino un modo permanente de operar todas sus funciones basado en el diagnóstico y la buena gestión de sus impactos directos e indirectos.

d. La responsabilidad social no es sólo para las empresas, sino que concierne a todas las organizaciones, públicas y privadas, con o sin fines de lucro, nacionales e internacionales (p. 9).

En este sentido, el tema de la responsabilidad social tiene un significado más profundo en el ámbito universitario. Desde sus inicios, ha sido considerado como el gerente de desarrollo y bienestar social, esta es su esencia. Además, al hablar de la responsabilidad social de la universidad, este concepto debe incluir actividades de promoción, investigación, gestión y previsión social, las principales actividades de formación integral, y otras funciones destinadas a fortalecer y comprobar la aplicación de la responsabilidad social; para lograr el bienestar general de una estructura armoniosa (Torres y Sánchez, 2014, pp. 69-105).

De acuerdo a los impactos que la universidad genera en el actuar cotidiano Vallaeys, De la Cruz y Sasia (2009, p. 18), indican que aquellos pueden ser agrupados en cuatro grandes rubros, como lo muestra la figura 2.

\section{Figura 2. Tipos de impactos universitarios}

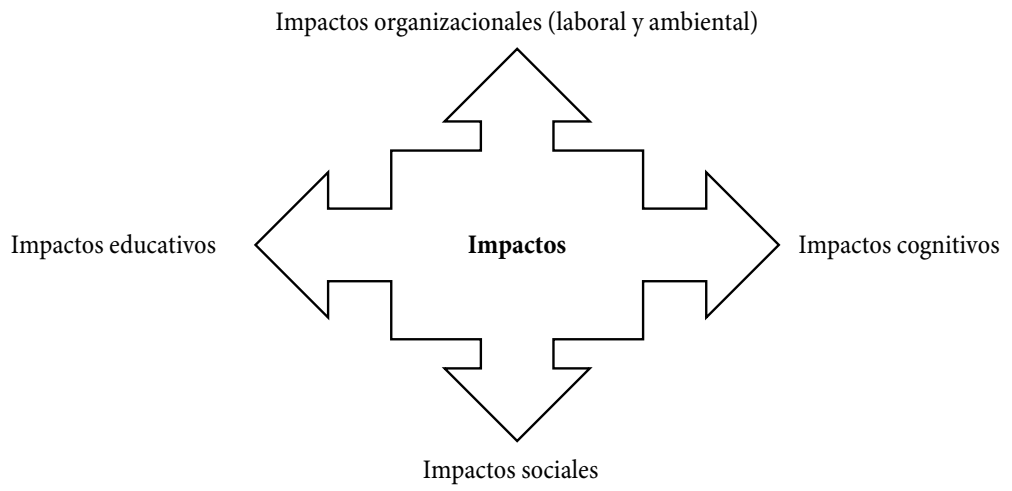

Fuente: Vallaeys, De la Cruz y Sasia (2009, p. 18). 
De igual manera, Vallaeys, De la Cruz, y Sasia (2009, p. 16) utilizan el concepto de stakeholders como un eje para el análisis y la evaluación del impacto del trabajo universitario. En este caso, permite identificar personal no docente y docente investigador, autoridades universitarias, estudiantes, proveedores, egresados, empleadores, competidores, comunidades, y organización local, colaboradora y estatal.

Según Yepes, Peñalosa, Marti, Díaz, y Gomez (2018, p. 8), el sistema de indicadores está compuesto por cinco grandes áreas, las cuales constituyen las funciones sustantivas de cualquier institución de educación superior y representan los compromisos y avances en términos de sostenibilidad que se quieren reportar, las cuales son:

Tabla 1. Estructura del Sistema de Indicadores

\begin{tabular}{|c|c|c|}
\hline Área & Aspecto general & Subaspectos \\
\hline \multirow{6}{*}{ 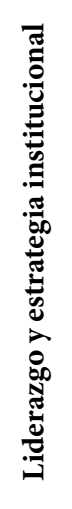 } & - Gobierno & $\begin{array}{l}\text { - Marco general, conflicto de interés y } \\
\text { equidad de género }\end{array}$ \\
\hline & - Estrategia institucional & $\begin{array}{l}\text { - Integración con la sostenibilidad, } \\
\text { grupos de interés y propósito }\end{array}$ \\
\hline & - Control de la operación & - Equipo y seguimiento \\
\hline & $\begin{array}{l}\text { - Reporte y rendición de } \\
\text { cuentas }\end{array}$ & - Reporte, verificación/comunicación \\
\hline & - Mejora e innovación & - Acciones y desarrollo \\
\hline & - Impacto & - Docencia, investigación y extensión \\
\hline \multirow{3}{*}{ 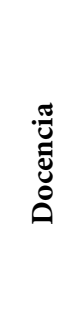 } & - Información general & $\begin{array}{l}\text { - Alcance y compromiso con la } \\
\text { sostenibilidad }\end{array}$ \\
\hline & - Formación responsable & $\begin{array}{l}\text { - Ética y ciudadanía, sostenibilidad } \\
\text { o responsabilidad social, liderazgo y } \\
\text { consumo responsable }\end{array}$ \\
\hline & - Formación pertinente & $\begin{array}{l}\text { - Contacto con la realidad y contexto } \\
\text { socioeconómico }\end{array}$ \\
\hline
\end{tabular}




\begin{tabular}{|c|c|c|}
\hline Área & Aspecto general & Subaspectos \\
\hline \multirow{2}{*}{ 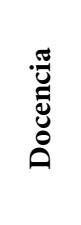 } & - Pluralidad en la formación & $\begin{array}{l}\text { - Interdisciplinariedad y } \\
\text { multiculturalidad }\end{array}$ \\
\hline & $\begin{array}{l}\text { - Integración de la formación } \\
\text { con los objetivos de desarrollo } \\
\text { sostenible (ODS) }\end{array}$ & $\begin{array}{l}\text { - Compromiso con la agenda de } \\
\text { desarrollo sostenible } 2030\end{array}$ \\
\hline \multirow{5}{*}{ 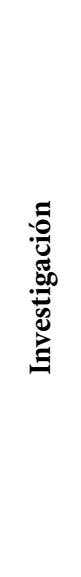 } & - Información general & $\begin{array}{l}\text { - Alcance y compromiso con la } \\
\text { sostenibilidad }\end{array}$ \\
\hline & - Investigación responsable & $\begin{array}{l}\text { - Ética y ciudadanía, sostenibilidad } \\
\text { o responsabilidad social, liderazgo y } \\
\text { consumo responsable }\end{array}$ \\
\hline & $\begin{array}{l}\text { - Vinculación a la } \\
\text { responsabilidad social o } \\
\text { sostenibilidad }\end{array}$ & $\begin{array}{l}\text { - Contacto con la realidad y contexto } \\
\text { socioeconómico }\end{array}$ \\
\hline & - Investigación colaborativa & $\begin{array}{l}\text { - Interdisciplinariedad y } \\
\text { multiculturalidad }\end{array}$ \\
\hline & $\begin{array}{l}\text { - Integración de la } \\
\text { investigación con los oDs }\end{array}$ & $\begin{array}{l}\text { - Compromiso con la agenda de } \\
\text { desarrollo sostenible } 2030\end{array}$ \\
\hline \multirow{6}{*}{ 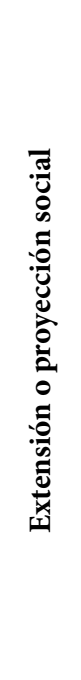 } & - Información general & $\begin{array}{l}\text { - Alcance y compromiso con la } \\
\text { sostenibilidad }\end{array}$ \\
\hline & $\begin{array}{l}\text { - Respuesta a las necesidades } \\
\text { de los beneficiarios o público } \\
\text { objetivo }\end{array}$ & $\begin{array}{l}\text { - Validación externa y canales de } \\
\text { atención }\end{array}$ \\
\hline & $\begin{array}{l}\text { - Articulación con actores } \\
\text { relevantes }\end{array}$ & $\begin{array}{l}\text { - Agendas de desarrollo local y global, } \\
\text { comunidad académica }\end{array}$ \\
\hline & $\begin{array}{l}\text { - Acceso y participación de } \\
\text { comunidades vulnerables o } \\
\text { minorías }\end{array}$ & $\begin{array}{l}\text { - Utilidad y aporte de las } \\
\text { intervenciones, selección y vinculación, } \\
\text { empoderamiento de las comunidades }\end{array}$ \\
\hline & - Cooperación pública/privada & $\begin{array}{l}\text { - Colaboración institucional y } \\
\text { proyectos para la sostenibilidad }\end{array}$ \\
\hline & $\begin{array}{l}\text { - Integración de la extensión } \\
\text { con los oDs }\end{array}$ & $\begin{array}{l}\text { - Compromiso con la agenda de } \\
\text { desarrollo sostenible } 2030\end{array}$ \\
\hline
\end{tabular}




\begin{tabular}{|c|c|c|}
\hline Área & Aspecto general & Subaspectos \\
\hline \multirow{5}{*}{ 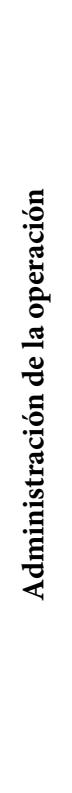 } & - Impacto ambiental & $\begin{array}{l}\text { - Energía, agua, biodiversidad, } \\
\text { emisiones, efluentes y residuos, } \\
\text { prevención, inversiones, cumplimiento, } \\
\text { suministro y transporte }\end{array}$ \\
\hline & - Aspectos laborales & $\begin{array}{l}\text { - Empleo, cumplimiento, relaciones, } \\
\text { salud y seguridad, participación, } \\
\text { inclusión y formación }\end{array}$ \\
\hline & - Respeto a los DD. HH. & $\begin{array}{l}\text { - Cumplimiento, seguimiento, } \\
\text { formación, verificación externa, } \\
\text { suministro, sanciones, libertad de } \\
\text { asociación, trabajo infantil y forzoso, } \\
\text { no discriminación, desplazamiento, } \\
\text { accesibilidad, bienestar }\end{array}$ \\
\hline & - Anticorrupción & $\begin{array}{l}\text { - Mecanismos, sistema de denuncias, } \\
\text { suministro, competencia leal }\end{array}$ \\
\hline & - Promoción y comunicación & $\begin{array}{l}\text { - Oferta académica, promoción y } \\
\text { mercadeo, privacidad y quejas y } \\
\text { reclamos }\end{array}$ \\
\hline
\end{tabular}

Fuente: "Sistema de indicadores de responsabilidad social universitaria" (Yepes, Peñalosa, Marti, Díaz y Gómez, 2018, p. 10).

Por otra parte, Vallaeys, De la Cruz y Sasia, (2009, pp. 33-34), identifican cuatro ejes de la RSU, derivados de los impactos empleados para el desarrollo de su metodología, acerca de la responsabilidad hacia el medio ambiente. Los cuatro ejes son el campus responsable, la formación profesional y ciudadana responsable, la generación y transmisión de conocimiento, y la participación social. El objetivo de estos de estos ejes es contribuir al desarrollo de una estrategia integral y sustentable para la responsabilización social universitaria.

El concepto de gestión de la responsabilidad social implica que la organización es consciente de todas las consecuencias de sus estrategias y acciones en el ámbito humano, social y medioambiental. Para promover 
el "desarrollo sostenible", la organización debe identificar su impacto en el medio ambiente (interno y externo) y resolver los problemas diagnosticados para que el funcionamiento normal de la organización deje de producir o pueda mitigar esos impactos; de tal manera que, alcance un nivel legal y socialmente aceptable (Vallaerys, De la Cruz y Sasia, 2009, p. 16).

La extensión universitaria conjuga elementos tan importantes como el trabajo de participación; la promoción humanística, comunitaria y ambientalmente sostenible; las alianzas estratégicas entre la sociedad civil, el estado, el sector privado y la academia; el trabajo multi, inter y transdisciplinario desde distintas líneas de acción y actores, en un proceso dialógico y de retroalimentación constante $[\ldots]$

El gran desafío para el siglo xxI es crear una ciudadanía ambientalmente sostenible, esto supone un proceso de formación en el que la responsabilidad social de las universidades adquiere un enorme protagonismo. No obstante, las nuevas tecnologías y el mercado han modificado la percepción del ciudadano en cuanto a la magnitud real del deber de las universidades respecto a la sociedad; esto ha causado que las universidades se dediquen ampliamente a la generación de recursos humanos cualificados y competentes para un mercado ocupacional restringido, mientras dejan de lado la participación en la formación y configuración de la ciudadanía (Chumaceiro, Hernández y Chirinos, 2016, pp. 4-6).

El proceso de desarrollo sustentable implica el cambio y el mejoramiento de los estilos de vida de la comunidad, el uso responsable y la gestión de los recursos, las nuevas formas de pensar y organizar la sociedad, los principios éticos, entre otros. Esto debe relacionarse con el proceso de percepción y desarrollo de actitudes sociales, de tal forma que los individuos adquieran las herramientas necesarias para tomar decisiones informadas en todos los aspectos de sus actividades sociales, interviniendo así en la mejora de su calidad de vida. En ese orden de ideas, la educación ambiental está compuesta por valores (difundidos en la verticalidad de las disciplinas), cambios en el comportamiento personal, e integración en los campos de la 
sociedad, la cultura, la economía, la raza y el orden poblacional (Peñafiel y Vallejo, 2018).

\section{Estudios previos}

A nivel internacional se identificaron múltiples estudios sobre la implementación de la responsabilidad social universitaria; Bermeo, Teijeiro, y García (2020, p. 12) afirman que las IEs evalúan su capacidad de gestión, en materia de RSU, mediante la preparación de informes de rendición de cuentas con la finalidad de ser eficientes, transparentes y ambientalmente responsables. Sin embargo, estos autores encontraron que son pocas las universidades públicas ecuatorianas que cumplen con los estándares requeridos para identificar y establecer sus políticas de sostenibilidad en cuestión de prácticas ambientales.

Por su parte, Yong-Ming, Yu-Long, Bin, Jia, y Yu-Ting (2020, pp. 3-5) realizan un análisis sobre la sostenibilidad de la educación superior en China según la comparación entre universidades chinas y estadounidenses, en donde comparan: la matrícula atendida, la preparación de sus académicos, los salarios que estos perciben, entre otras variables. Sin abordar el tema de responsabilidad social, su estudio revela que las universidades de China han presentado un gran crecimiento en los últimos años en esos aspectos, pero sigue existiendo una brecha frente a las universidades estadounidenses.

Es frecuente escuchar hablar de la responsabilidad social, pero pocos saben que este tema, tan asociado al sector empresarial, también puede tener una aplicación en el ámbito de las instituciones de educación superior; ya que, sin importar si estas tienen o no un fin lucrativo, pueden aplicar los mismos principios y prácticas en su gestión, para abordar la Rsu. De acuerdo con El Economista (2019, p. 1), en el caso particular de la Universidad Anáhuac México, este tema también ha sido estudiado desde hace varios años; además, se ha llevado a la práctica a través del Comité de RSU y Sustentabilidad, conformado por miembros de diversas áreas, escuelas y facultades, con la intención de que el tema de responsabilidad 
social no sólo se vea reflejado por el cumplimiento de la misión educativa, sino que sea parte del modelo de gestión de la institución.

\section{Metodología}

El presente trabajo de investigación Análisis de la responsabilidad social universitaria: un estudio comparativo en Latinoamérica corresponde a una investigación cualitativa, puesto que busca analizar el problema mediante la interpretación y composición de la responsabilidad social universitaria en las Instituciones de Educación Superior. Teniendo en cuenta los objetivos de esta investigación, se realizará un estudio documental para informar cómo las universidades sujetas de estudio han implementado el tema medioambiental en su plan de desarrollo institucional. De tal manera, se describe el objetivo de estudio en detalle y se transforma la información disponible en un cuerpo coherente de ideas.

Los sujetos de estudio de este artículo son la Universidad Autónoma de Baja California México y la Universidad Santo Tomas en Bogotá, Colombia. Estas forman parte de la Red de Cuerpos Académicos en Responsabilidad Social Empresarial (Recarse), un acuerdo de colaboración suscrito por los cuerpos académicos: Organizaciones Sociales y Productivas Regionales - CA - 33, de la Facultad de Ciencias Económicas Administrativas de la Universidad Autónoma del Carmen; Investigación Educativa Desarrollo y Competitividad de las Organizaciones y Entidades Económicas, de la Facultad de Contaduría y Administración de la Universidad Veracruzana; y la Asociación Colombiana de Facultades de Contaduría Pública. Asimismo, participaron los cuerpos académicos de Empresarialidad Social y Microfinanzas, y el de Micro, pequeñas y medianas empresas, ambos registrados en la Facultad de Contaduría de Administración de la Universidad Autónoma de Baja California (Tijuana, México).

Inicialmente se intentaba comparar a todas las universidades que conforman la Recarse, pero, debido al corto tiempo y a la falta de información 
disponible en los sitios web oficiales de las IEs que la conforman, se decidió solo hacerlo de la universidad de Baja California y la de Bogotá.

Las fuentes secundarias en las que se centra este estudio son el Plan de Gestión Ambiental: un compromiso de la USTA con el planeta de la Universidad Santo Tomas, el Plan de Desarrollo Institucional de la Universidad Autónoma de Baja California, Principles for Responsible Management Education y el Sistema de indicadores de responsabilidad social universitaria (Yepes et al., 2018).

Para el análisis y presentación de los resultados se utilizó la técnica de análisis comparativo de contenido con base en seis categorías: el uso responsable de la energía eléctrica, el gasto responsable del agua, el compromiso con el reciclaje, el manejo de residuos, la biodiversidad y por último las emisiones.

\section{Análisis y resultados}

A partir de la revisión documental, se propone el presente sistema de indicadores capaz de medir las áreas referentes al ámbito de protección del medio ambiente como parte de la RsU. El objetivo de este sistema es proporcionar a las universidades participantes en este estudio una herramienta básica para cuantificar y valorar los resultados alcanzados. Para ello, se realiza un análisis de las estrategias implementadas por estas instituciones para el logro de su objetivo sobre medio ambiente en el marco de la Rsu. Esto permitirá conocer y reforzar las áreas de oportunidad con mayor precisión y fiabilidad.

Desde la perspectiva del uso responsable de la electricidad, se puede ver que tanto la Universidad Autónoma de Baja California como la Universidad Santo Tomas están implementando buenas prácticas en el uso responsable de la energía eléctrica. No obstante, la usTa ha puesto en marcha estrategias más específicas para crear conciencia en este aspecto; tanto en las sedes principales como en el campus universitario se han activado diferentes campañas para sensibilizar a la comunidad universitaria (tabla 2). 
Tabla 2. Uso responsable de la energía eléctrica

\begin{tabular}{|l|l|l|}
\hline \multicolumn{1}{|c|}{ Universidad } & \multicolumn{1}{|c|}{ Objetivo } & \multicolumn{1}{c|}{ Estrategia } \\
\hline $\begin{array}{l}\text { Universidad } \\
\text { Autónoma de } \\
\text { Baja California }\end{array}$ & $\begin{array}{l}\text { Proponer e implementar } \\
\text { medidas para la optimización y el } \\
\text { buen uso del recurso energético } \\
\text { en la universidad. }\end{array}$ & $\begin{array}{l}\text { Campaña de sensibilización. } \\
\text { Uso de lámparas led. } \\
\text { Instalación de generador } \\
\text { fotovoltaico. }\end{array}$ \\
\hline $\begin{array}{l}\text { Universidad } \\
\text { Santo Tomás }\end{array}$ & $\begin{array}{l}\text { Proponer e implementar } \\
\text { medidas para la optimización y el } \\
\text { buen uso del recurso energético } \\
\text { en la universidad. }\end{array}$ & $\begin{array}{l}\text { Producción de energía } \\
\text { renovable en las propias } \\
\text { instalaciones. }\end{array}$ \\
\hline
\end{tabular}

Fuente: elaboración propia con base en Gómez (2018), Ortiz (2017), Treviño (2017) y Velásquez (2017).

Sumado a esto, la UABC y la USTA han implementado la mediación para el ahorro de agua: por un lado, la UABC crea tecnología de jardinería en seco a través de áreas de mantenimiento y áreas verdes; por el otro, la USTA realiza actividades de publicidad y capacitación sobre el uso responsable del agua. Las dos universidades están comprometidas con el cuidado del agua por eso han implementado campañas, con el fin de sensibilizar a toda la comunidad universitaria (tabla 3 ).

Tabla 3. Gasto responsable del agua

\begin{tabular}{|l|l|l|}
\hline \multicolumn{1}{|c|}{ Universidad } & \multicolumn{1}{|c|}{ Objetivo } & \multicolumn{1}{c|}{ Estrategia } \\
\hline $\begin{array}{l}\text { Universidad } \\
\text { Autónoma de } \\
\text { Baja California }\end{array}$ & $\begin{array}{l}\text { Propiciar el ahorro de agua para } \\
\text { su uso eficiente, el tratamiento de } \\
\text { aguas residuales y el uso de agua } \\
\text { morada. }\end{array}$ & $\begin{array}{l}\text { Zonas de mantenimiento. } \\
\text { Creación de áreas verdes } \\
\text { a través de la técnica de } \\
\text { xerojardinería. }\end{array}$ \\
\hline & $\begin{array}{l}\text { Proponer e implementar } \\
\text { medidas para asegurar el } \\
\text { aprovisionamiento de agua } \\
\text { potable, la optimización y el } \\
\text { buen uso del recurso hídrico } \\
\text { Santo Tománás }\end{array}$ & $\begin{array}{l}\text { Crear campañas de } \\
\text { sensibilización y capacitación } \\
\text { frente al consumo } \\
\text { responsable del agua y el } \\
\text { manejo de aguas residuales. }\end{array}$ \\
\hline
\end{tabular}

Fuente: elaboración propia con base en Gómez (2018), Ortiz (2017), Treviño (2017) y Velásquez (2017). 
De acuerdo con su compromiso con el reciclaje, las dos universidades implementaron campañas de reciclaje. Sin embargo, la UABC especificó aún más sus campañas; por ejemplo, mediante la instalación de contenedores de basura y pegatinas en los botes de basura de los campus de la universidad, para facilitar la identificación del tipo de basura correspondiente a cada contenedor, mejorando así la eficiencia del reciclaje (tabla 4).

Tabla 4. Compromiso con el reciclaje

\begin{tabular}{|c|c|c|}
\hline Universidad & Objetivo & Estrategia \\
\hline $\begin{array}{l}\text { Universidad } \\
\text { Autónoma de } \\
\text { Baja California }\end{array}$ & $\begin{array}{l}\text { Contribuir con el cuidado del } \\
\text { medio ambiente gestionando } \\
\text { la tarea de reciclaje de plástico } \\
\text { y aluminio; la instalación de } \\
\text { depósitos de basura en todos los } \\
\text { campus, en los que se dividen } \\
\text { los residuos por su material, } \\
\text { para facilitar el reciclaje. }\end{array}$ & $\begin{array}{l}\text { Campaña "Cero residuos". } \\
\text { Reciclaje de pastico y } \\
\text { aluminio. } \\
\text { Calcomanías en los botes de } \\
\text { basura para la selección de } \\
\text { esos residuos. }\end{array}$ \\
\hline $\begin{array}{l}\text { Universidad } \\
\text { Santo Tomás }\end{array}$ & $\begin{array}{l}\text { Generar comportamientos } \\
\text { responsables frente a los } \\
\text { residuos cotidianos de las } \\
\text { instalaciones y asegurar que } \\
\text { una gran parte de esos residuos } \\
\text { entren al proceso de reciclaje. }\end{array}$ & $\begin{array}{l}\text { Campañas de reciclaje con la } \\
\text { ayuda del personal de Casa } \\
\text { Limpia. } \\
\text { Instalación de puntos } \\
\text { ecológicos para mejorar la } \\
\text { separación en la fuente de } \\
\text { residuos ordinarios. }\end{array}$ \\
\hline
\end{tabular}

Fuente: elaboración propia con base en Gómez (2018), Ortiz (2017), Velásquez (2017) y Treviño (2017).

En cuanto a la gestión de residuos, tanto la UABC como la USTA han implementado nuevas estrategias para el uso adecuado de los residuos, como lo son la implementación de programas y capacitación para el personal encargado de estos residuos. Por último, la USTA instaló nuevas áreas de almacenamiento de residuos como lo indica la tabla 5. 
Tabla 5. Manejo de residuos

\begin{tabular}{|l|l|l|}
\hline Universidad & \multicolumn{1}{|c|}{ Objetivo } & \multicolumn{1}{c|}{ Estrategia } \\
\hline $\begin{array}{l}\text { Universidad } \\
\text { Autónoma de } \\
\text { Baja California }\end{array}$ & $\begin{array}{l}\text { Asegurar el manejo y el tratamiento } \\
\text { de residuos sólidos (en especial } \\
\text { de los residuos peligrosos) con } \\
\text { base en la normatividad aplicable } \\
\text { yenfocados en su reducción y } \\
\text { valorización. }\end{array}$ & $\begin{array}{l}\text { Capacitación para el } \\
\text { personal relacionada } \\
\text { con el manejo correcto y } \\
\text { segregación de los residuos } \\
\text { que se derivan de las } \\
\text { operaciones y actividades. }\end{array}$ \\
\hline Universidad & $\begin{array}{l}\text { Establecer e implementar las } \\
\text { estrategias necesarias para reducir } \\
\text { la generación de residuos peligrosos } \\
\text { (Registro de Generadores de } \\
\text { Residuos o Desechos Peligrosos), } \\
\text { regular el manejo y disposición } \\
\text { final de los mismos en la } \\
\text { universidad con el fin de proteger la } \\
\text { salud y el ambiente. }\end{array}$ & $\begin{array}{l}\text { Capacitación para el } \\
\text { personal encargado de } \\
\text { estos residuos. } \\
\text { Incluir al interior de la } \\
\text { universidad infraestructura } \\
\text { para la recolección de } \\
\text { residuos peligrosos en } \\
\text { el marco de las políticas } \\
\text { nacionales de posconsumo. }\end{array}$ \\
\hline
\end{tabular}

Fuente: elaboración propia con base en Gómez (2018), Ortiz (2017), Treviño (2017) y Velásquez (2017).

En materia de biodiversidad, la UABC y la UsTA prometen proteger la naturaleza a través de la implementación de programas de sensibilización y campañas, entre otras herramientas. Para ello, la UABC impulsó la implementación del proyecto de huerta FCQI Pachamama, con el fin de contribuir al cuidado del medio ambiente y así darle un mejor uso a la tierra (tabla 6).

Tabla 6. Biodiversidad

\begin{tabular}{|c|c|c|}
\hline Universidad & Objetivo & Estrategia \\
\hline $\begin{array}{l}\text { Universidad } \\
\text { Autónoma de } \\
\text { Baja California }\end{array}$ & $\begin{array}{l}\text { Contribuir al cuidado del medio } \\
\text { ambiente y acercar a la comunidad } \\
\text { universitaria a la naturaleza y lograr } \\
\text { una seguridad alimentaria, teniendo } \\
\text { un acceso a los alimentos que sean } \\
\text { cosechados. }\end{array}$ & $\begin{array}{l}\text { Promover el proyecto } \\
\text { de Huerto FCQI } \\
\text { Pachamama }\end{array}$ \\
\hline
\end{tabular}




\begin{tabular}{|l|l|l|}
\hline Universidad & \multicolumn{1}{|c|}{ Objetivo } & \multicolumn{1}{c|}{ Estrategia } \\
\hline & $\begin{array}{l}\text { Establecer acciones para conservar y } \\
\text { proteger la fauna y flora presente en } \\
\text { las instalaciones de la universidad, } \\
\text { promoviendo un pensamiento ético } \\
\text { ambiental que fomente el cuidado de } \\
\text { la naturaleza y el fortalecimiento de } \\
\text { la dimensión ambiental al interior de } \\
\text { Universidad } \\
\text { Santo Tomás } \\
\text { lanstitución y en todas sus funciones } \\
\text { universitarias. }\end{array}$ & $\begin{array}{l}\text { Implementar el Plan } \\
\text { de Comunicación } \\
\text { Ambiental } \\
\text { (capacitaciones, } \\
\text { eventos, campañas, } \\
\text { publicaciones, etc.) }\end{array}$ \\
\hline
\end{tabular}

Fuente: elaboración propia con base en Gómez (2018), Ortiz (2017), Treviño (2017) y Velásquez (2017).

Finalmente, en el tema de las emisiones, las dos universidades están comprometidas con la adecuada gestión y atención al medio ambiente, y con realizar campañas para la comunidad universitaria. Sin embargo, la USTA ha establecido una estrategia más profunda, gestionando adecuadamente las emisiones e implementando un proyecto para el uso del material vegetal producido por la poda de áreas verdes, de los lodos y los sobrenadantes de la trampa de grasa. El objetivo de este proyecto es darles un buen uso a esas emisiones, como la fertilización a través de procesos de composta (tabla 7).

Tabla 7. Emisiones

\begin{tabular}{|l|l|l|}
\hline \multicolumn{1}{|c|}{ Universidad } & \multicolumn{1}{|c|}{ Objetivo } & \multicolumn{1}{|c|}{ Estrategia } \\
\hline & $\begin{array}{l}\text { Promover campañas instituciones para } \\
\text { asegurar el uso adecuado de los espacios } \\
\text { clasificados como libres de humo, } \\
\text { promoviendo el ahorro de energía, } \\
\text { Universidad } \\
\text { Autónoma de } \\
\text { Baja California } \\
\text { su vez el congestionamiento vehicular, } \\
\text { contribuyendo a la disminución de } \\
\text { emisiones de } \mathrm{CO}_{2}\end{array}$ & $\begin{array}{l}\text { Implementar } \\
\text { el programa } \\
\text { Eco-cimarrón. }\end{array}$ \\
\hline
\end{tabular}




\begin{tabular}{|l|l|l|}
\hline Universidad & \multicolumn{1}{|c|}{ Objetivo } & \multicolumn{1}{c|}{ Estrategia } \\
\hline & $\begin{array}{l}\text { Establecer las estrategias para el } \\
\text { manejo adecuado de los residuos } \\
\text { sólidos ordinarios generados en la } \\
\text { universidad, con el fin de prevenir y } \\
\text { minimizar los riesgos para la salud y el } \\
\text { ambiente, sensibilizando a la comunidad } \\
\text { Saniversidaria frente a la gestión adecuada } \\
\text { de residuos y sus impactos ambientales. }\end{array}$ & $\begin{array}{l}\text { Imprentar el proyecto } \\
\text { en el campus } \\
\text { aprovechando las } \\
\text { áreas verdes de este. }\end{array}$ \\
\hline
\end{tabular}

Fuente: elaboración propia con base en Gómez (2018), Ortiz (2017) y Treviño (2017), Velásquez (2017).

\section{Conclusión}

En las conclusiones alcanzadas se propone la creación de nuevas políticas y estrategias que permitan brindar soluciones desde el origen de los residuos, como la reducción, el reciclaje y la reutilización; dichas estrategias se armonizan con la preocupación mundial sobre el mejor aprovechamiento de los recursos y la contaminación de los espacios públicos. En ese orden de ideas, Alfie (2003, p. 92) propone concretar un programa integral frente al medio ambiente, con políticas de cuidado y educación ambiental que reorienten la misión de las instituciones, donde quede claro el compromiso institucional por avanzar en el conocimiento e investigación ambiental. Se trata de fomentar la transformación de valores y prácticas y acceder a realizar un plan guía que cuide y mejore el medio ambiente con la aprobación de políticas de cuidado y educación ambiental.

Para Mata, Claudia Zúñiga, Brenes, Carrillo, Charpentier, Hernández y María Zúñiga:

La educación ambiental se plantea como el sendero educativo que facilita la transmisión de claves culturales para que el individuo y los grupos sociales se adapten de forma responsable, en su sentido ecológico, al medio en que viven y se desarrollan. Es la formación que permite conocer y reconocer 
las interacciones entre lo que hay de "natural" y de "social" en su entorno; $\mathrm{y}$ de actuar en este entorno sin deteriorar el equilibrio que los procesos naturales han desarrollado, tendiendo a lograr una calidad de vida idónea para el desarrollo de la vida humana (Mata et al., 2003).

Una conciencia ambiental en la sociedad universitaria determina las decisiones en ese ámbito; debido a eso, analizar, diagnosticar y desarrollar herramientas para potenciar dicha conciencia ambiental constituye un paso básico a la hora de diseñar e implementar planes y programas eficientes de educación ambiental. Además, aquella

[...] tiene un potencial extraordinario, tanto por la fase educativa en la que se encuentran como por la proximidad de incorporación en el mundo laboral, en el que realmente se presentarán dilemas y conflictos sobre cuestiones ambientales que habrán de resolver aplicando la conciencia y educación ambiental que hayan adquirido (Peñafiel y Vallejo, 2018).

Tanto la Universidad Autónoma de Baja California como la Universidad Santo Tomas han implantado varias estrategias para el cuidado del medio ambiente; concientizando a las comunidades educativas para el cuidado y la protección de aquel. Cabe resaltar que estas universidades deben comenzar a unir nuevas estrategias pedagógicas para una buena formación ambiental en todos los aspectos; invitando e incluyendo a los administradores, docentes y alumnos a tener un buen uso en las áreas correspondientes al cuidado del medio ambiente. Por el momento, solo utilizando prácticas antiguas, que les han funcionado, pero no han tenido en cuenta las nuevas prácticas que la sociedad ha puesto en funcionamiento en el cuidado del medio ambiente.

Por último, se propone que las dos universidades busquen nuevas estrategias o actualicen las ya implementadas, con el fin de gestionar bien los campos relacionados con el ámbito de la protección ambiental; de tal forma que, las estrategias utilizadas en la universidad sean también utilizadas por la comunidad. Finalmente, se propone poner en práctica el mejoramiento de aquellas o encontrar una nueva forma de involucrar a toda la comunidad universitaria en la protección del medio ambiente; ya que, las instituciones 
de educación superior son factores fundamentales para la reacción eficaz del medio ambiente, en ellas no solo se tiene en cuenta la comunidad educativa, sino la sociedad en la que cada ser humano crece, actúa e interactúa.

\section{Referencias}

Alfie, M. (2003). Medio ambiente y universidad: retos y desafíos ambientales en la Universidad Autónoma Metropolitana-Azcapotzalco. El Cotidiano, 86-92. https://www.redalyc.org/pdf/325/32512210.pd

Bermeo Pacheco, J. A., Teijeiro-Álvarez, M. M., y García-Álvarez, M. T. (2020). Sustainable Development in the Economic, Environmental, and Social Fields of Ecuadorian Universities. Sustainability, 12(18), 7384. https://doi.org/10.3390/ su12187384

Bustos, C. e Inciarte, A. (2012). Dimensión comunitaria de la responsabilidad social universitaria. Opción, 28(68), 367-379. https://www.redalyc.org/articulo. oa?id $=31025437008$

Chumaceiro, A., Hernández, J. y Chirinos, E. (2016). Responsabilidad social universitaria, desarrollo sostenible y ciudadanía ambiental. Cuadernos de RSO, 4(1), 53-64. https://www.researchgate.net/publication/313037924_ Responsabilidad_Social_Universitaria_desarrollo_sostenible_y_ciudadania_ambiental

Estrella Suárez, M. V. y González Vázquez, A. (2017). Desarrollo sustentable: Un nuevo mañana. Grupo Editorial Patria.

Franco, M., Medina, R., Torres, L., Velázquez, K., Valencia, M., y Valencia, A. (2017). La responsabilidad social universitaria en la actual sociedad del conocimiento. Un acercamiento necesario. Medisur, 15(6). http://scielo.sld.cu/ scielo.php?script=sci_arttext\&pid=S1727-897X2017000600006\&fbclid=IwA R3HR1mKV3g9HObbsqn69jQOgub0oYnxUPpdn2kwOiiyScIE57dY8Enh5sM

Gaete, R. (2015). El concepto de responsabilidad social universitaria desde la perspectiva de la alta dirección. Cuadernos de Administración, 31(53). https:// cuadernosdeadministracion.univalle.edu.co/index.php/cuadernos_de_administracion/article/view/20 
González, L., Méndez, E., García, J. y Arguello, F. (2017). La responsabilidad social universitaria. El cumplimiento de los fines de la universidad. Espacios Públicos, 20(50). https://www.redalyc.org/jatsRepo/676/67656569008/html/index.html

Gómez, A. (2018). Implementa UABC manejo adecuado de los residuos. http:// gaceta.uabc.mx/notas/academia/implementa-uabc-manejo-adecuado-delos-residuos

Larrouyet, M. C. (2015). Desarrollo sustentable: Origen, evolución y su implementación para el cuidado del planeta [Trabajo final integrador, Universidad Nacional de Quilmes]. http://ridaa.unq.edu.ar/handle/20.500.11807/154

Mata, A., Zúñiga, C., Brenes, O., Carrillo, M., Charpentier, C., Hernández, L., y Zúñiga, M. (2003). Estrategias innovadoras para la formación inicial de educadores en el campo ambiental. http://hdl.handle.net/20.500.12337/2488

Ojeda, J., y Álvarez, D. (2015). Responsabilidad social en las universidades: antecedentes, trayectorias y perspectivas. Coepes, 4(12). http://www.revistacoepesgto.mx/revistacoepes12/responsabilidad-social-en-las-universidadesantecedentes-trayectorias-y-perspectivas

Ordoñez, L., y Werner, R. (2019). La educación para el desarrollo sostenible en la universidad boliviana. Percepciones del profesorado. Teoría de la Educación, 31(1), 149-173. http://dx.doi.org/10.14201/teri.19037

Peñafiel, M., y Vallejo, A. (2018). Educación ambiental en las universidades, retos y desafíos ambientales. Revista DELOS Desarrollo Local Sostenible. https:// www.eumed.net/rev/delos/32/magaly.html

Torres, A. y Sánchez, L. (2014). La responsabilidad social universitaria desde su fundamentación teórica. Libre Empresa, 11(1). https://doi.org/10.18041/16572815/libreempresa.2014v11n1.3014

Treviño, D. (23 de octubre de 2017). Generan huerto orgánico en la Facultad DeCiencias Químicas e Ingeniería. GacetaUABC. http://gaceta.uabc.mx/notas/academia/ generan-huerto-organico-en-la-facultad-de-ciencias-quimicas-e-ingenieria Universidad Autónoma de Baja California. (2017). Coordinación de proyectos de gestión. http://www.uabc.mx/GestionAmbiental/

Vallaeys, F. (s. f.). ¿Qué es la Responsabilidad Social Universitaria? http://creasfile. uahurtado.cl/RSU.pdf 
Vallaeys, F. (s. f.). Breve marco teórico de responsabilidad social universitaria. https://www.econo.unlp.edu.ar/frontend/media/86/10986/10ec2d7e617a62 eba0696821196efeae.pdf

Vallaeys, F. (2014). La responsabilidad social universitaria: Un nuevo modelo universitario contra la mercantilización. Revista Iberoamericana de Educación Superior, 5(12). http://www.scielo.org.mx/scielo.php?script=sci_arttext\&pid $=$ S2007-28722014000100006

Vallaeys, F., De la Cruz, C., y Sasia, P. (2009). Responsabilidad social universitaria Manual de primeros pasos. Mc Graw Hill. https://www.academia.edu/14028570/ Manual_de_primeros_pasos_en_Responsabilidad_Social_Universitaria

Velásquez, V. (2017). Planes de Gestión Ambiental. Universidad Santo Tomás. https://gestionambiental.usta.edu.co/index.php/nuestra-gestion

Yepes, G., Peñalosa, L., Martí, J., Díaz, N. Forigua, G. y Gómez, D. (2018). Sistema de Indicadores de Responsabilidad Social Universitaria para el Informe de Progreso - PRME. Corporación Red Local del Pacto Global en Colombia. https://isbn. cloud/9789585684201/sistema-de-indicadores-de-responsabilidad-socialuniversitaria-para-el-informe-de-progreso-prme/

Yong-Ming, H., Yu-Long, P., Bin, R., Jia, K. y Yu-Ting, S. (2020). Analysis on the Higher Education Sustainability in China Based on the Comparison between Universities in China and America. Sustainability, 12(2). https://doi. org/10.3390/su12020573

Zepeda, J., Andrade, A. y González, J. (2017). Origen y evolución del concepto de desarrollo sustentable. Realidad económica, 19(52). https://www.realidadeconomica.umich.mx/ 